\section{Accuracy Evaluation of EPL and ETAR Algorithms in the Treatment Planning Systems using CIRS Thorax Phantom}

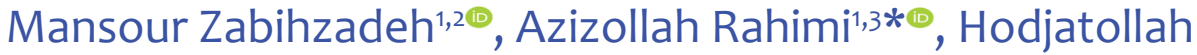 \\ Shahbazian ${ }^{4}$, Sasan Razmjoo ${ }^{4}$, Seyyed Rabie Mahdavi ${ }^{5}$
}

\begin{abstract}
Background: It is recommended for each set of radiation data and algorithm that subtle deliberation is done regarding dose calculation accuracy. Knowing the errors in dose calculation for each treatment plan will result in an accurate estimate of the actual dose achieved by the tumor.

Objective: This study aims to evaluate the equivalent path length (EPL) and equivalent tissue air ratio (ETAR) algorithms in radiation dose calculation.

Material and Methods: In this experimental study, the TEC-DOC 1583 guideline was used. Measurements and calculations were obtained for each algorithm at specific points in thorax CIRS phantom for 6 and $18 \mathrm{MVs}$ and results were compared.
\end{abstract}

Results: In the EPL, calculations were in agreement with measurements for 27 points and differences between them ranged from $0.1 \%$ to $10.4 \%$ at $6 \mathrm{MV}$. The calculations were in agreement with measurements for 21 points and differences between them ranged from $0.4 \%$ to $13 \%$ at $18 \mathrm{MV}$. In ETAR, calculations were also in consistent with measurements for 21 points, and differences between them ranged from $0.1 \%$ to $9 \%$ at $6 \mathrm{MV}$. Moreover, for $18 \mathrm{MV}$, the calculations were in agreement with measurements for 17 points and differences between them ranged from $0 \%$ to $11 \%$.

Conclusion: For the EPL algorithm, more dose points were in consistent with acceptance criteria. The errors in the ETAR were $1 \%$ to $2 \%$ less than the EPL. The greatest calculation error occurs in low-density lung tissue with inhomogeneities or in high-density bone. Errors were larger in shallow depths. The error in higher energy was more than low energy beam.

Citation: Zabihzadeh M, Rahimi A, Shahbazian H, Razmjoo S, Mahdavi SR. Accuracy Evaluation of EPL and ETAR Algorithms in the Treatment Planning Systems using CIRS Thorax Phantom. J Biomed Phys Eng. 2021;11(4):483-496. doi: 10.31661/jbpe.v0i0.1097.

\section{Keywords}

Algorithms; Dose Calculation Error; Lung Tissue; Inhomogeneities; Radiotherapy; Treatment Planning Systems; Radiation Dosage

\section{Introduction}

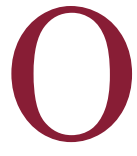

ne of the most important issues to analyze a treatment plan in radiotherapy is ensuring that sufficient dose reaches the target (tumor) to control its growth and prevent radiation from reaching the normal tissues surrounding the tumor. Before performing any radiation therapy, considering the characteristics of the anatomy, clinical findings, facilities and devices, a suitable treatment planning is conducted for the patient [1]. Nowadays, it has not been practically possible to get a direct measurement of the distribution of the three-dimensional
${ }^{1} \mathrm{PhD}$, Department of Medical Physics, Faculty of Medicine, Ahvaz Jundishapur University of Medical Sciences,

Ahvaz, Iran

${ }^{2} \mathrm{PhD}$, Department

of Clinical Oncology,

Faculty of Medicine,

Golestan Hospital, Ahvaz

Jundishapur University

of Medical Sciences,

Ahvaz, Iran

${ }^{3} \mathrm{PhD}$, Department of

Radiology, Paramedi-

cal school, Hamadan

University of Medical Sci

ences, Hamadan, Iran

${ }^{4} \mathrm{MD}$, Department

of Clinical Oncology,

Faculty of Medicine,

Golestan Hospital, Ahvaz

Jundishapur University

of Medical Sciences,

Ahvaz, Iran

${ }^{5} \mathrm{PhD}$, Department of

Medical Physics, Faculty

of Medicine, Iran Univer-

sity of Medical Sciences,

Tehran, Iran

*Corresponding author:

Azizollah Rahimi

Department of Medical

Physics, Faculty of Medi-

cine, Ahvaz Jundishapur

University of Medical

Sciences, Ahvaz, Iran

E-mail: azizrahimi91@

gmail.com

Received: 1 January 2019 Accepted: 15 April 2019 
(3D) dose delivered to the patient. Therefore, treatment planning should be based on computational models. Even if direct measuring is possible, planning will be more practical and simpler based on computational models. The predicted dose by a computational method should be equal to the actual absorbed dose in the patient's body, as precisely as possible. The delivered dose to the tumor volume should be close to the prescribed dose. Some organs have critical tolerance dose levels that should not be exceeded, otherwise serious side effects may occur. Besides, for further understanding of biological response mechanisms in radiotherapy, accurate calculation of the dose is essential. In addition, the biological response of cells to radiation is totally nonlinear; thus, little errors in predicted dose make big mistakes in biological response [2]. It should be noted that dose calculation method is not the only source of difference between the distributions of predicted dose and delivered dose, but the beam shaping and the patient position, the movements and changing position of patient and output inconstancy of the system are substantial factors in causing errors. Today, various treatment-planning algorithms have been developed to correct tissue heterogeneity in treatment planning systems. Choosing an appropriate treatment-planning algorithm in radiotherapy is very crucial, especially for tissues with high heterogeneity, such as the lungs, oral cavity, teeth, nasal cavity, and bones with different physical and radiological characteristics. Although in different algorithms, corrections for heterogeneity have been too much considered, each algorithm has its own limitations for accurate dose estimation [3]. Each treatment-planning software has some errors in the dose calculations. These errors are more seen especially in heterogeneous areas. For each radiation data set and each algorithm, it is recommended to make accurate calculations regarding the accuracy of dose calculations [4]. So far, numerous studies have been conducted to examine treatment-plan- ning algorithms [5-16]. The first techniques for dose calculation are developed based on empirical formulas [17]. In these techniques, primary and scattered components of radiation are considered separately because they have different physical behaviors within the matter. The primary component defines the distribution of transferred energy by the interaction of the primary photon in the matter, and the secondary component predicts the result of a series of subsequent interactions distributing energy away from the primary interaction. Many new treatment planning systems use physical density information (mass density) or electron density for each tissue to correct dose calculations in heterogeneous tissues. This information is derived from $\mathrm{CT}$ numbers conversion into the electron density data sheets in treatment planning systems [18]. The error of dose calculation in each treatment planning system will be computed by physicians and physicists with a fairly accurate estimation of actual dose delivered to the tumor. It is shown that a dose difference of up to $7 \%$ can be consequential clinically and a standard deviation of a determined dose of $5 \%$ or more can affect the response to the treatment [19-20]. To assess the efficacy of a treatment planning system, the International Atomic Energy Agency (IAEA) has issued a practical guideline (IAEATECDOC 1583) based on TRS-430 [21]. In this study, the procedure of responding both Equivalent Path Length (EPL) and Equivalent Tissue Air Ratio (ETAR) computational algorithms was examined. EPL methods determine the correction factor by tracking the beam directly from the primary radiation source to the point of interaction [22]. More complicated techniques, such as the ETAR method, use the electron density data obtained from using the weighted average density in the irradiated volume to determine the correction factors [2324]. However, the use of these correction factors may also lead to differences of more than $10 \%$ with the measured dose for some geometry. The two methods of EPL and ETAR are 
defined as below, based on the dimensions of density information used.

\section{Material and Methods}

This work is an experimental study.

\section{Equivalent Path Length (EPL) method}

Calculations in this method are based only on the densities in the direction of the primary paths of photons. According to the densities obtained from CT scan images and the combination of information, this method can be used in 3D treatment plans. However, since only the densities in the primary path of beam are considered, this method is considered as a one-dimensional algorithm. In this method, the dose is attained at a point by the information obtained from the central axis of the beam, such as Tissue-Air Ratio (TAR), Tissue-Maximum Ratio (TMR), Tissue-Phantom Ratio (TPR) or Percent Depth Dose (PDD), and off-axis ratios [25]. The desired depth $\mathrm{Z}$ is obtained by equation 1 based on the average of the densities in the path to the geometric depth $\mathrm{z}$ :

$$
z^{\prime}=\frac{1}{\rho_{w}} \int_{0}^{z} \rho\left(z^{\prime \prime}\right) d z^{\prime \prime}
$$

$\rho_{w}$ is the density of water and $\rho\left(\mathrm{Z}^{\prime \prime}\right)$ is the local density of the tissue, which in most cases are extracted from the $\mathrm{CT}$ images. Correction values can be obtained by equation 2 [2] from four methods included of effective attenuation, TAR ratio, effective $\mathrm{SSD}$, and isodose shift [26-29]:

$C F=e^{-\mu\left(z^{\prime}-z\right)}=\frac{\operatorname{TAR}\left(A \cdot z^{\prime}\right)}{\operatorname{TAR}(A \cdot z)}=\frac{P D D\left(A \cdot z^{\prime} \cdot S S D\right)}{\operatorname{PDD}(A \cdot z \cdot S S D)}\left(\frac{S S D+z^{\prime}}{\operatorname{SSD}+z}\right)^{2}=\frac{\operatorname{PDD}\left(A \cdot z-n\left(z-z^{\prime}\right) \cdot S S D\right)}{\operatorname{PDD}(A \cdot z \cdot S S D)}(2)$

where, $\mathrm{n}$ is obtained empirically.

The EPL method almost models the primary dose changes, except in the cases of intense charged particle disequilibrium. The scattered dose reaching the given point depends on both the location and size of heterogeneity [2].

Equivalent Tissue Air Ratio (ETAR) method

This method was developed by Sontag and
Cunningham in 1978 to be used in computer system [23-24]. Using the scaling density theory [30], the TAR value in a field with radius $\mathrm{A}_{\mathrm{r}}$ at depth $\mathrm{z}$ in a matter with density $\rho$ relative to water is equal to TAR $\left(\rho \mathrm{A}_{\mathrm{r}} ; \rho \mathrm{z}\right)$. The ETAR correction factor is defined as follows:

$$
C F=\frac{T A R\left(\rho A_{r} ; \rho z\right)}{\operatorname{TAR}\left(A_{r} ; z\right)}
$$

This correction factor is valid only for a completely homogeneous medium similar to water with non-unit density. By changing $\rho \mathrm{A}_{\mathrm{r}}$ to $\tilde{\rho}_{\mathrm{r}} \mathrm{A}_{\mathrm{r}}$ and $\rho \mathrm{z}$ to $\tilde{\rho}_{\mathrm{z}} \mathrm{z}$, this formula can be applied to a heterogeneous medium. $\tilde{\rho}_{\mathrm{r}}$ and $\tilde{\rho}_{\mathrm{z}}$ are effective densities that $\tilde{\rho}_{\mathrm{r}}$ and $\rho_{\mathrm{z}}$ are obtained by the weighted average of the densities in the irradiated volume and averaging the density values in the primary beam path (such as EPL methods) [2].

\section{A. Phantom}

In this experimental study, functionality of two treatment planning systems - RT dose (version 1) with EPL computational algorithm, and CorePlan (version 3.5.0.5) with ETAR computational algorithm - using CIRS thorax phantom model 002LFC as shown in Figure 1 and the recommended tests in IAEA-TECDOC 1583 were assessed. This CIRS phantom has materials for simulating different body tissues whose density specifications are given in

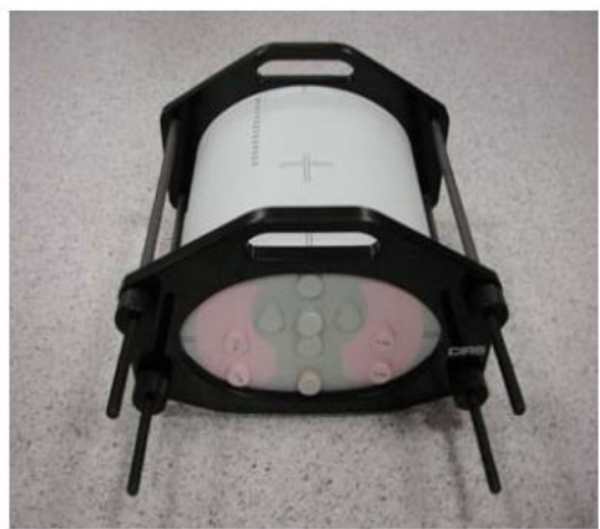

Figure 1: Thorax Phantom (computerized imaging reference systems (CIRS); model 002 LFC). 
Table 1. The phantom is elliptical, and almost modeling the thorax shape of human body that length and width are $30 \mathrm{~cm}$; in addition, height is $20 \mathrm{~cm}$. This phantom is made of materials that fairly models soft tissue, bone, and lung with a $1 \%$ error within the range of $50 \mathrm{keV}$ $25 \mathrm{MeV}$. The phantom consists of two completely symmetric halves. Half of the phantom $(15 \mathrm{~cm})$ is divided into 12 sections, and each of them, except the first one, has $1 \mathrm{~cm}$ thickness. The other half is integrated and has cylinders equivalent to tissue, lung, and bone that are removable, and it also ionization chamber and point dose measurements to be placed in different phantom areas (see Figure 2). The phantom surface has a scale facilitating the phantom alignment [21].
B. CT scan

For the treatment planning, Siemens Somatom Emotion 16 slice CT scanner was used to take CT images of the phantom. The density curve entered into the treatment planning system was compared with the density values obtained for phantom. The images were acquired in $130 \mathrm{KV}$ and with slice thickness of $3 \mathrm{~mm}$.

\section{Dosimetry}

The Farmer chamber (PTW-30010) dosimeter and PTW-UNIDOS electrometer were used to detect and read ionization.

\section{Irradiation}

Phantom irradiation was performed by VARIAN CLINAC $2100 \mathrm{C}$ accelerator system at 6

Table 1: Certified density reference materials for the computerized imaging reference systems (CIRS) phantom.

\begin{tabular}{ccc} 
& & $\begin{array}{c}\text { Density } \\
\left(\mathbf{g} / \mathbf{c m}^{3}\right)\end{array}$ \\
\hline Lung & 0.21 \\
\hline Bone & 1.60 \\
\hline Muscle & 1.06 \\
\hline Adipose & 0.96 \\
\hline Plastic water (body) & 1.04 \\
\hline & & \\
\hline & & 1 \\
\hline
\end{tabular}

Figure 2: Labelling of holes: Plug $1=$ water equivalent, plug 2 = muscle substitute, plug 3 = syringe filled with water, Plug $4=$ adipose substitute, plug 5 = water equivalent, plug 6 lung substitute, plug $7=$ empty to represent air, Plug $8 \& 9=$ lung substitute, plug $10=$ bone substitute.

\begin{tabular}{cc}
$\begin{array}{c}\text { Electron Density Per } \\
\mathbf{c m}^{\mathbf{3}} \mathbf{\times} \mathbf{1 0}^{\mathbf{2 3}}\end{array}$ & $\begin{array}{c}\text { Electron Density relative } \\
\text { to water }\end{array}$ \\
\hline 0.69 & 0.207 \\
\hline 5.03 & 1.506 \\
\hline 3.48 & 1.042 \\
\hline 3.17 & 0.949 \\
\hline 3.35 & 1.003
\end{tabular}

MV energy and $18 \mathrm{MV}$ energy. Due to the lack of MLC, out of 8 recommended tests by TECDOC -1583, 7 tests were carried out for defined holes inside the phantom (CIRS Model 002 LFC) as shown in Figure 2; MLC's test number 5 was not conducted. In treatmentplanning software, grid size $5 \mathrm{~mm}$ was used for dose calculation. The purpose, application and structure of the tests are as follows:

Test 1 - Confirmation of computations for reference fields: This test aimed to validate the calculations for the reference field based on the relative densities obtained from $\mathrm{CT}$ data. A $10 \times 10 \mathrm{~cm}^{2}$ field was used with gantry and collimator angles of $0^{\circ}$. The ionization chamber was placed at points 3,9 and 10. The dose of $200 \mathrm{cGy}$ was delivered to point 3.

Test 2 - Confirmation of computations for 
lack of scattering in tangential fields: This test aimed to validate the computations when there was no scattering in tangential field. A field of $15 \times 10 \mathrm{~cm}^{2}$ was created with a $45^{\circ}$ wedge and $90^{\circ}$ gantry angle. The ionization chamber was placed at point 1; in addition, the dose of 200 cGy was delivered to point 1 . The reference point was point 1 .

Test 3 - Confirmation of computations for block fields: A $14 \times 14 \mathrm{~cm}^{2}$ field was created by a $45^{\circ}$ collimator angle. The primary field changed into a $10 \times 10 \mathrm{~cm}^{2}$ field by the blocks. The amount of $200 \mathrm{cGy}$ was delivered to point 3 . The reference point was point 3 .

Test 4 - Confirmation of computations of the dose received from a field and the total dose of four fields in a four-field technique: The total dose of $200 \mathrm{cGy}$ was delivered to point 5 with four fields with gantry angels of $0^{\circ}, 180^{\circ}, 90^{\circ}$, and $270^{\circ}$. For gantry angles of $0^{\circ}$ and $180^{\circ}$, a field of $15 \times 10 \mathrm{~cm}^{2}$ was created, and a field of $15 \times 8 \mathrm{~cm}^{2}$ was created for gantry angles of $90^{\circ}$ and $270^{\circ}$. Dose values reaching to points 5,6 , and 10 were obtained for each field and sum of doses was also acquired. Point 5 was considered as the reference point.

Test 5 (test 6 in TECDOC 1583) - Confirmation of computations in irregular fields with block in the center of the field: A $20 \times 10 \mathrm{~cm}^{2}$ field was created. The gantry angle was $45^{\circ}$ and the collimator angle was $0^{\circ}$. A lead shield blocked the field and a final field of $12 \times 6 \mathrm{~cm}^{2}$ was created. The dose of 200 cGy was delivered to point 3 . In addition, Dose values were recorded at points 7 and 10 . Point 3 was considered as the reference point.

Test 6 (test 7 in TECDOC 1583) - Confirmation of computations in three fields, with two pair wedge fields with asymmetric collimation: Three fields with gantry angles of $0^{\circ}, 90^{\circ}$, and $270^{\circ}$ were created. The isocenter was placed at point 3 . The dose of $200 \mathrm{cGy}$ was delivered to point 5 as the reference point. For the other two fields, gantry was placed at angles of $90^{\circ}$ and $270^{\circ}$. For gantry of $90^{\circ}$, the field was set to $Y=10 \mathrm{~cm}, X_{1}=0 \mathrm{~cm}$, and $X_{2}$
$=6 \mathrm{~cm}$. Wedge of $30^{\circ}$ was placed on the field. Gantry angle was then placed at $270^{\circ}$, and irradiation was done again with the same field and wedge. In all three fields, the doses were obtained at point 5 . Point 5 was considered as the reference point.

Test 7 (test 8 in TECDOC 1583) - Confirmation of computations in collimator and couch rotation: Three fields were created at different gantry and collimator angles. First, a field of 4 $\times 16 \mathrm{~cm}^{2}(\mathrm{X}=16 \mathrm{~cm}, \mathrm{Y}=4 \mathrm{~cm})$ was formed with a $90^{\circ}$ gantry and a 330 collimator. The dose of 200 cGy was delivered to point 5 . Then, without changing the field size, gantry was placed at $270^{\circ}$ and the rotation angle of collimator was placed at $30^{\circ}$. The dose of 200 cGy was delivered to point 5 and for the third field, the field was set to $4 \times 4 \mathrm{~cm}^{2}$. For the third field, the field was set to $4 \times 4 \mathrm{~cm}^{2}$. Gantry was placed at $30^{\circ}$ and collimator was also placed at $30^{\circ}$. The couch was rotated $270^{\circ}$. Point 5 was considered as the reference point. For all tests, measurements were performed 3 times by ionization chamber for each point, and then the mean value was obtained. Comparison of measurements and calculations was carried out in 26 situations in phantom. In addition, five measurements were related to the sum of values obtained from the fields associated with a specific test at an intended point. A total of 31 computations and measurements were obtained for each treatment planning system with particular energy.

\section{E. Data analysis}

The evaluation of the difference between measured and calculated values was obtained using the following formula:

$$
\operatorname{Error}[\%]=100 \times\left[\left(D_{\text {calc }}-D_{\text {meas }}\right) / D_{\text {meas.ref }}\right]
$$

$\mathrm{D}_{\text {meas }}$ is the measured dose at the intended point; $\mathrm{D}_{\text {calc }}$ is the calculated dose at the same point, and $\mathrm{D}_{\text {meas, ref }}$ is the measured dose at the reference point. The use of the reference point, according to TECDOC 1540, is due to the small number of measured points available in 
Mansour Zabihzadeh, et al

the CIRS phantom [31].

ment, calculation and agreement criteria related to each test for EPL and ETAR algorithms at $6 \mathrm{MV}$ energy are given in Table 2.

Besides, the differences between the results

Results for 6 MV energy

of measurement and calculation for both al-

The differences in the results of measure-

gorithms are shown in Figure 3. Comparison

Table 2: Comparison of differences between measurement results with equivalent path length (EPL) \& equivalent tissue air ratio (ETAR) algorithms at $6 \mathrm{MV}$.

\begin{tabular}{|c|c|c|c|c|c|}
\hline $\begin{array}{c}\text { Case. Point. } \\
\text { Field }\end{array}$ & $\begin{array}{c}\text { EPL Deviation } \\
(\%)\end{array}$ & $\begin{array}{c}\text { ETAR Deviation } \\
(\%)\end{array}$ & $\begin{array}{l}\text { Agreement } \\
\text { Criteria (\%) }\end{array}$ & EPL Result & ETAR Result \\
\hline C1.P3 & -0.3 & -0.8 & \pm 2 & $P$ & $P$ \\
\hline C1.P9 & -2.7 & -2.2 & \pm 4 & $P$ & $P$ \\
\hline C1.P10 & -0.9 & -1.9 & \pm 3 & $P$ & $P$ \\
\hline C2.P1 & 1.2 & 6.5 & \pm 3 & $P$ & FAIL \\
\hline C3.P3 & -1.6 & -1.1 & \pm 3 & $P$ & $P$ \\
\hline C4.P5.F1 & -1.6 & 0.7 & \pm 2 & $P$ & $\mathrm{P}$ \\
\hline C4.P5.F2 & -0.4 & 2.9 & \pm 3 & $P$ & $P$ \\
\hline C4.P5.F3 & 2.3 & 0.8 & \pm 3 & $P$ & $P$ \\
\hline C4.P5.F4 & 0.8 & 2.1 & \pm 3 & $P$ & $P$ \\
\hline C4.P5.SUM & 0.3 & 1.6 & \pm 3 & $P$ & $P$ \\
\hline C4.P6.F1 & -0.4 & -0.1 & \pm 4 & $P$ & $P$ \\
\hline C4.P6.F2 & 3.3 & 6.0 & \pm 3 & FAIL & FAIL \\
\hline C4.P6.F3 & -2.7 & -0.5 & \pm 4 & $P$ & $P$ \\
\hline C4.P6.F4 & 8.0 & 7.3 & \pm 3 & FAIL & FAIL \\
\hline C4.P6.SUM & 2.2 & 3.2 & \pm 3 & $P$ & FAIL \\
\hline C4.P10.F1 & -3.0 & -0.7 & \pm 3 & $P$ & $P$ \\
\hline C4.P10.F2 & -0.4 & 1.5 & \pm 4 & $P$ & $P$ \\
\hline C4.P10.F3 & -1.1 & -1.6 & \pm 3 & $P$ & $P$ \\
\hline C4.P10.F4 & 0.1 & 1.7 & \pm 4 & $P$ & $P$ \\
\hline C4.P10.SUM & -1.1 & 0.2 & \pm 3 & $P$ & $P$ \\
\hline C5.P3 & -0.8 & 1.5 & \pm 3 & $P$ & $P$ \\
\hline C5.P7 & 6.0 & 9.3 & \pm 5 & FAIL & FAIL \\
\hline C5.P10 & -9.8 & -8.4 & \pm 5 & FAIL & FAIL \\
\hline C6.P5.F1 & -1.1 & 0.6 & \pm 2 & $P$ & $P$ \\
\hline C6.P5.F2 & -0.4 & 2.7 & \pm 4 & $P$ & $P$ \\
\hline C6.P5.F3 & 0.5 & 1.7 & \pm 4 & $P$ & $P$ \\
\hline C6.P5.SUM & -0.4 & 1.7 & \pm 3 & $P$ & $P$ \\
\hline C7.P5.F1 & -1.3 & 4.8 & \pm 3 & $P$ & FAIL \\
\hline C7.P5.F2 & -0.5 & 5.6 & \pm 3 & $P$ & FAIL \\
\hline C7.P5.F3 & 1.0 & 5.4 & \pm 3 & $P$ & FAIL \\
\hline C7.P5.SUM & -0.3 & 5.5 & \pm 3 & $P$ & FAIL \\
\hline
\end{tabular}

EPL: Equivalent path length, ETAR: Equivalent tissue air ratio 


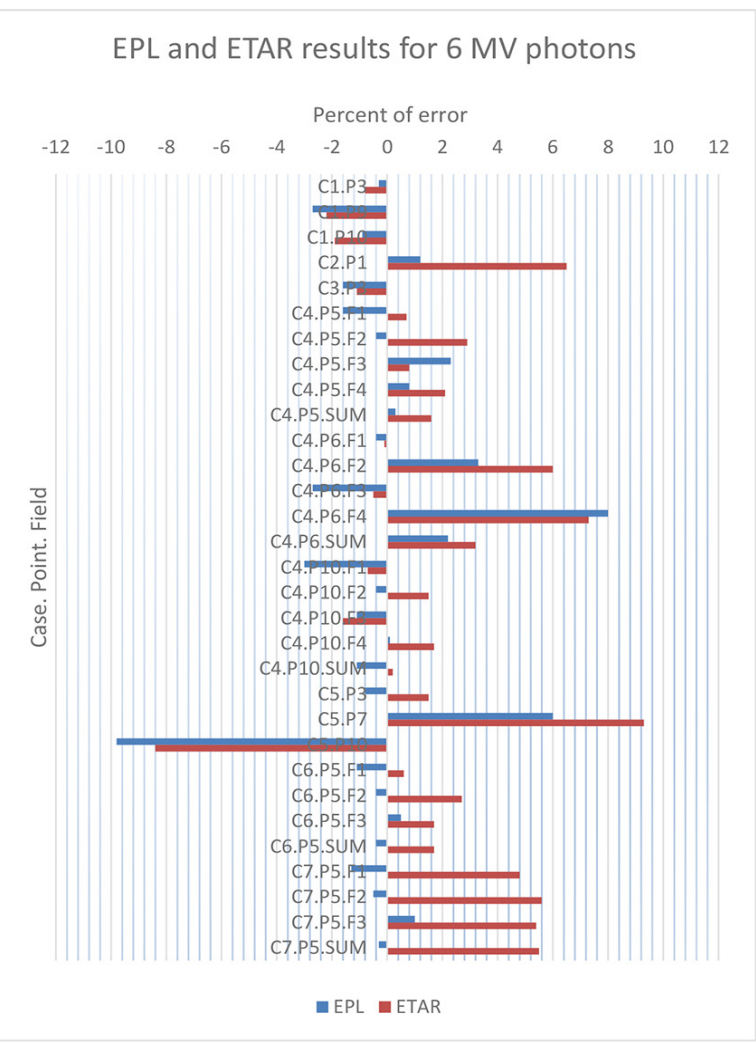

Figure 3: Equivalent path length (EPL) \& equivalent tissue air ratio (ETAR) results for $6 \mathrm{MV}$ photons.

of measurements and calculations was carried out at 26 points of phantom. Five calculations and measurements were related to the sum of values obtained from the fields associated with a specific test at an intended point.

\section{Results for 18 MV energy}

The differences resulting from the measurement, the calculation and agreement criteria for the various tests in EPL and ETAR algorithms at $18 \mathrm{MV}$ energy are given in Table 3.

Besides, in Figure 4, the difference between the results of measurement and calculation for both algorithms is shown. Comparison of measurements and calculations was carried out at 26 points of phantom. Five calculations and measurements were related to the sum of the values obtained from the fields associated with a specific test at an intended point.

\section{Discussion}

A total of 31 calculations and measurements were compared in Table 2. For the EPL algorithm, the calculations in 27 cases were in line with the agreement criteria and passed the tests. At 4 points, the algorithm could not pass tests. For the ETAR algorithm, the calculations in 21 cases were in line with the agreement criteria and passed the tests. In 10 cases, the algorithm could not be at acceptable level. The ETAR algorithm could not pass test 2, while the EPL algorithm passed the test. The objective of this test was to confirm the calculation for the effect of scattering in tangential fields. The calculated value by the ETAR algorithm in this test was $6.5 \%$ higher than the measured value. It seems that the ETAR algorithm calculates the dose more than the real value when some parts of the radiation field expand to the surrounding air around the tissue (like the situation in the tangential treatment of breast cancer). In the EPL algorithm, two failed cases were related to test 4 , which was the four-field box test. In test 4 , the calculated values at point 6 in the fields with gantry angles of $0^{\circ}$ and $180^{\circ}$ were close to the measured values, and the errors of both algorithms were acceptable in these two fields. The calculated values at point 6 in two fields with gantry angles of $90^{\circ}$ and $270^{\circ}$ were more than the measurements. For the EPL algorithm, the differences in lung tissue varied from $3.3 \%$ to $8 \%$, depending on the depth of the considered point. In field with gantry angles of $90^{\circ}$, the beam from the left side was entered the phantom and the point 6 was located on the right side of the phantom. In this case, the calculated dose was $3.3 \%$ higher than the measured value. In the field with gantry angles of $270^{\circ}$, the beam was also entered the phantom from the right side and point 6 was also on the right side. In this case, the difference between the calculation and the measurement was $8 \%$.

For the ETAR algorithm in test 4 , in the field with gantry angles of $90^{\circ}$ at point 6 , the calculated value was $6 \%$ greater than the mea- 
Mansour Zabihzadeh, et al

sured value. In the field with gantry angles of $270^{\circ}$ at point 6 , the calculated value was $7.3 \%$ higher than the measured value. The other two cases, in which the differences in the measurement and calculation values were more than the agreement criteria were related to test 5 (point 7 that were located within the right lung tissue at the radiation field edge, and point 10 that was placed within the bone tissue under the shield). This test was used to confirm the calculations in irregular fields with block in the center of the field. In the EPL algorithm,

Table 3: Comparison of differences between measurement results with equivalent path length (EPL) \& equivalent tissue air ratio (ETAR) algorithms at $18 \mathrm{MV}$.

\begin{tabular}{|c|c|c|c|c|c|}
\hline $\begin{array}{l}\text { Case. Point. } \\
\text { Field }\end{array}$ & $\begin{array}{c}\text { EPL Deviation } \\
(\%)\end{array}$ & $\begin{array}{l}\text { ETAR Deviation } \\
(\%)\end{array}$ & $\begin{array}{l}\text { Agreement } \\
\text { Criteria (\%) }\end{array}$ & EPL Result & ETAR Result \\
\hline C1.P3 & -0.4 & -0.5 & \pm 2 & $P$ & $P$ \\
\hline C1.P9 & -7.5 & -5.5 & \pm 4 & FAIL & FAIL \\
\hline C1.P10 & -3.2 & -3.8 & \pm 3 & FAIL & FAIL \\
\hline C2.P1 & 0.6 & 4.7 & \pm 3 & $P$ & FAIL \\
\hline C3.P3 & -0.8 & -0.5 & \pm 3 & $P$ & $P$ \\
\hline C4.P5.F1 & -1.2 & -0.6 & \pm 2 & $P$ & $P$ \\
\hline C4.P5.F2 & -1.0 & 0.4 & \pm 3 & $P$ & $P$ \\
\hline C4.P5.F3 & 1.4 & 1.7 & \pm 3 & $P$ & $P$ \\
\hline C4.P5.F4 & -0.1 & 0.0 & \pm 3 & $P$ & $P$ \\
\hline C4.P5.SUM & -0.2 & 0.4 & \pm 3 & $P$ & $P$ \\
\hline C4.P6.F1 & -3.7 & -1.2 & \pm 4 & $P$ & $P$ \\
\hline C4.P6.F2 & 4.0 & 4.5 & \pm 3 & FAIL & FAIL \\
\hline C4.P6.F3 & -7.2 & -4.7 & \pm 4 & FAIL & FAIL \\
\hline C4.P6.F4 & 8.5 & 6.5 & \pm 3 & FAIL & FAIL \\
\hline C4.P6.SUM & 0.4 & 1.4 & \pm 3 & $P$ & $P$ \\
\hline C4.P10.F1 & -4.7 & -4.1 & \pm 3 & FAIL & FAIL \\
\hline C4.P10.F2 & -1.9 & -0.2 & \pm 4 & $P$ & $P$ \\
\hline C4.P10.F3 & -5.0 & -5.0 & \pm 3 & FAIL & FAIL \\
\hline C4.P10.F4 & -1.6 & -0.2 & \pm 4 & $P$ & $P$ \\
\hline C4.P10.SUM & -3.4 & -2.4 & \pm 3 & FAIL & $P$ \\
\hline C5.P3 & 1.7 & 0.5 & \pm 3 & $P$ & $P$ \\
\hline C5.P7 & 11.2 & 11.0 & \pm 5 & FAIL & FAIL \\
\hline C5.P10 & -12.8 & -10.9 & \pm 5 & FAIL & FAIL \\
\hline C6.P5.F1 & -0.7 & 0.9 & \pm 2 & $P$ & $P$ \\
\hline C6.P5.F2 & -1.0 & 2.8 & \pm 4 & $P$ & $P$ \\
\hline C6.P5.F3 & -0.3 & 2.1 & \pm 4 & $P$ & $P$ \\
\hline C6.P5.SUM & -0.7 & 1.9 & \pm 3 & $P$ & $P$ \\
\hline C7.P5.F1 & 0.8 & 4.5 & \pm 3 & $P$ & FAIL \\
\hline C7.P5.F2 & 1.9 & 5.7 & \pm 3 & $P$ & FAIL \\
\hline C7.P5.F3 & 2.5 & 5.3 & \pm 3 & $P$ & FAIL \\
\hline C7.P5.SUM & 1.7 & 5.2 & \pm 3 & $P$ & FAIL \\
\hline
\end{tabular}

EPL: Equivalent path length, ETAR: Equivalent tissue air ratio 
Accuracy Evaluation of Algorithms in TPS

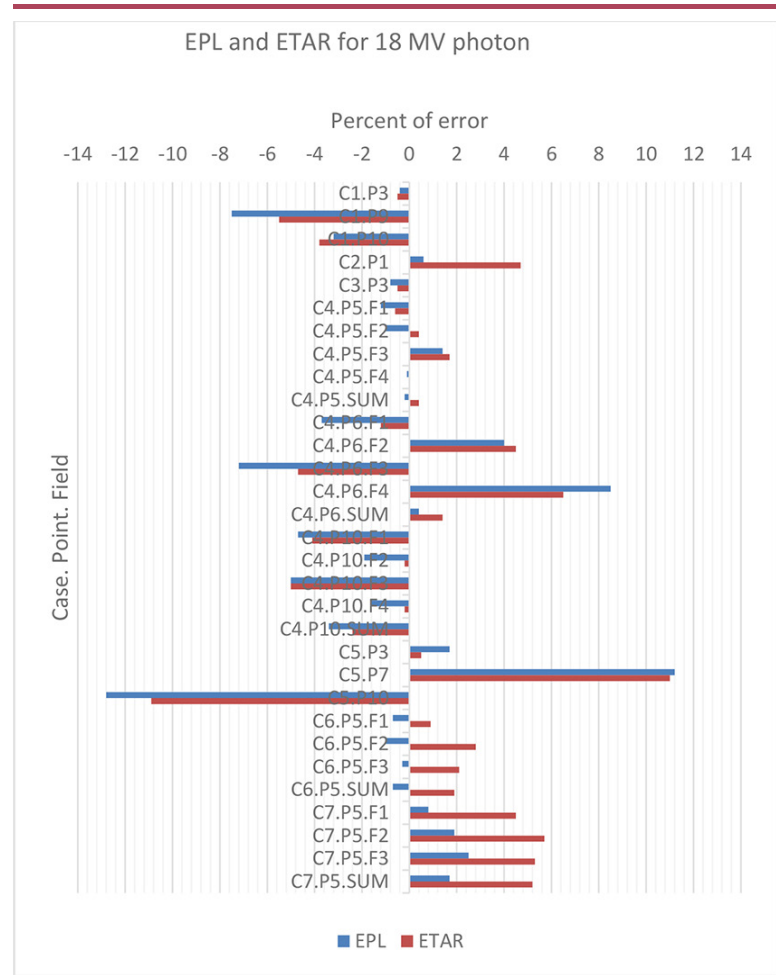

Figure 4: Equivalent path length (EPL) \& equivalent tissue air ratio (ETAR) results for $18 \mathrm{MV}$ photon.

the calculated dose in the lung tissue was $6 \%$ higher than the measured value. In the ETAR algorithm, the calculated dose value was 9.3 $\%$ higher than the measured value. Therefore, at this point it was seen that the ETAR algorithm's error was more than the EPL algorithm's error.

Both the EPL and the ETAR algorithms computed the amount of doses in an acceptable range and the bone area inside the field that the doses at point 10 in Test 1 and the first and third fields in Test 4 were in the acceptable error range. However, both algorithms demonstrated that the doses in bone area under the shield were significantly less than the measured values $(-9.8 \%$ and $-8.4 \%$, respectively), in which the EPL algorithm's error was $1.5 \%$ higher than the ETAR algorithm's error. This test showed that both algorithms estimated the dose resulting from scattering of bone tissue (when bone was shielded) less than the actual dose. Both algorithms passed test 6 . Test 7 was used to confirm the calculations in the mode of collimator and bed rotation. The EPL algorithm passed test 7 , but the ETAR algorithm failed to pass the test. The calculated value by the ETAR algorithm was higher than the measured value by more than $5.6 \%$.

A total of 31 calculations and measurements were compared with each other. For the EPL algorithm, in 21 situations, the calculations were in the range of agreement criteria and passed the tests. The algorithm could not be acceptable in 10 situations. For the ETAR algorithm in 17 situations, the calculations were in line with the agreement criteria and passed the tests. In 14 situations, the algorithm was not able to reach the acceptable level. In test 1 at point 9 within the lung and outside the radiation field, the calculated value in the EPL algorithm was 7.5\% and in the ETAR algorithm was $5.5 \%$ lower than the measured value. In this case, the error of ETAR algorithm was $2 \%$ less than the EPL algorithm's error. In test 1 , the calculated value of the dose at point 10 within the equivalent tissue to the bone, inside the radiation field, in the EPL algorithm was $3.2 \%$ and in the EATR algorithm was $3.3 \%$ lower than the measured value. Based on comparison carried out between C1.P10 and C4.P10.F1 (for field of $10 \times 10$ with field of $10 \times 15)$ to calculate the dose at point 10 using both algorithms, it could be seen that by increasing the field size, the difference between the measurement and the calculation was greater. Thus for bone tissues, the number of occurred interactions between radiation and tissue was estimated by both algorithms less than the real values. This growth in the difference was greater in the EPL algorithm than in the ETAR algorithm (in the ETAR algorithm, by increasing the field size, the difference between measurements and calculations increased from $-3.8 \%$ to $-4.1 \%$, while in the EPL algorithm, the difference increased from $-3.2 \%$ to $-4.7 \%$ ).

EPL algorithm passed test 2 at both energies. 
In the ETAR algorithm in test 2, the calculated value was $4.7 \%$ higher than the measured value. It seems that in tangential fields, when some parts of the radiation field extended to the surrounding air around the tissue (e.g. the situation in the tangential treatment of breast), this algorithm calculated the dose value more than the real value. In this case, the error decreased by increasing the energy (comparing test 2 at both energies of 6 and 18 for the ETAR algorithm).

In test 4 in field with gantry angles of $90^{\circ}$ at point 6 , the calculated value in EPL was $4 \%$ and in ETAR was $4.5 \%$ higher than the measured value. In field with gantry angles of $270^{\circ}$ at point 6 , the calculated value in EPL was $8.5 \%$ and in ETAR was $6.5 \%$ higher than the measured value. In the second field of test 4 , at point 6 , the ETAR algorithm failed with more errors than the EPL algorithm. In the fourth field of test 4 , at point 6 the EPL algorithm failed with more errors than the ETAR algorithm. The test suggested that at $18 \mathrm{MV}$ energy as at $6 \mathrm{MV}$ energy, in low density tissues at shallow depths, the EPL algorithm had more errors than the ETAR algorithm. However, by increasing the depth, the error of EPL algorithm was less than that of the ETAR algorithm and the difference between the calculation and the measurement decreased. In addition, this reduction was less for the ETAR algorithm. In fact, in the ETAR algorithm, compared with the EPL algorithm, the method of calculating the dose was less dependent on depth.

In the EPL algorithm in fields with gantry angles of $0^{\circ}$ and $180^{\circ}$ in test 4 , point 6 was placed outside the field. In these two cases, only the scattered dose reached point 6 . The calculated values in these two cases were less than measured values. Although the calculated value was acceptable in the field of $0^{\circ}$ with an error of $-3.7 \%$, in field of $180^{\circ}$ the calculated dose was measured to be $7.2 \%$ lower than the measured value. In the ETAR algorithm, the doses at this point for fields of $0^{\circ}$ and $180^{\circ}$ were $-1.2 \%$ and $-4.7 \%$ less than the measured value, respectively. In both fields of $0^{\circ}$ and $180^{\circ}$, both algorithms calculated the doses below the measured values, but apparently, the ETAR algorithm calculated the doses with less error. The greater difference between the calculated and measured values in the field of $180^{\circ}$ compared to the field of $0^{\circ}$ might be due to the heterogeneity of bone tissue in the beam path in the field of $180^{\circ}$. There was no bone tissue heterogeneity in the field of $0^{\circ}$ in the beam path. However, for lung tissue outside of the beam path, the calculated dose value was estimated to be less than the measured value.

In test 4 , in the field of $0^{\circ}$ at point 10 (bone tissue equivalent), the calculated values of the dose in the EPL algorithm was $4.7 \%$ and in the ETAR algorithm was $4.1 \%$ lower than the measured values. In fact, both algorithms at high energy (18 MV) calculated the number of interactions within the bone tissue less than the real values. In this test, in the field of $180^{\circ}$, the calculated dose by both algorithms was $5 \%$ less than the measured value. Reduction of error by increasing the depth, in the ETAR algorithm was more than in the EPL algorithm. Furthermore, in both algorithms, the error reduction process in terms of depth at high-density material (bone) was lesser than that in low-density tissues (lung).

In test 5 at point 7 within the lung, which was located inside the field, the calculated values in EPL and ETAR were, respectively, $11.2 \%$ and $11 \%$ more than the measured value. As seen, the behavior of both algorithms was equal towards this test. For the point 10 within the bone under the shield, the calculated values in EPL and ETAR were, respectively, 12.8\% and $10.9 \%$ lower than the measured value. Both algorithms passed test 6 .

The EPL algorithm passed test 7 , but the ETAR algorithm failed to pass the test. The calculated value by the ETAR algorithm was higher than the measured value by more than $5.7 \%$.

Both algorithms passed more tests at low energy than at high energy. So that at $6 \mathrm{MV}$ 
energy, the EPL and ETAR algorithms passed 27 and 21 tests, respectively, and at $18 \mathrm{MV}$ energy, they passed 21 and 14 tests, respectively. By increasing the energy of the beam, the absolute value of the difference between calculation and measurement also increased for both the lung and bone tissues.

Other studies also showed that these algorithms responded better at lower energies [7, $15,32]$.

At $6 \mathrm{MV}$ energy, in the second field of test 4 at point 6 , although both algorithms failed to pass the test, the ETAR algorithm failed with more errors than the EPL algorithm. However, in the fourth field of test 4 at point 6 , both algorithms failed to pass the test, the EPL algorithm failed with more errors than the ETAR algorithm. This test declared that in low-density tissues at lower depths, the EPL algorithm showed more errors compared to the ETAR algorithm. Nevertheless, by increasing the depth, the error of the EPL algorithm was less than the ETAR algorithm's error and the difference between the calculation and measurement decreased. In addition, this reduction was less in the ETAR algorithm. In fact, the method of calculating the dose in the ETAR algorithm was less dependent on the depth than the EPL algorithm.

At both energies in both algorithms for the points inside the field within the lung tissue (point 7 in test 5), doses were estimated to be greater than the measured values. Other studies also indicated that correction-based algorithms calculated the dose value in tumors inside the lung more than measured values; thus, they delivered less than expected doses to the tumor [5-6, 8, 10]. Engelsman et al., study in 2001 on two Pencil Beam (PB) algorithms with one-dimensional density corrections (modified Batho and EPL algorithm) showed that these algorithms might estimate the amount of doses in areas with low density up to $20 \%$ more than the measured value [12]. Furthermore, in 2003, the evaluation of the EPL algorithm at the lung / tumor boundary by De
Jaeger et al., suggested that the algorithm estimated the dose value $10 \%$ more than the measured value [14]. In addition, Aarup et al., in evaluation of Pencil Beam Convolution (PBC) algorithms in lung tissue demonstrated that the algorithms estimated the dose value more than the actual value [16]. Rutonjski et al., studied two algorithms of CMS XiO software. In the correction-based algorithm for test 5 at point 7 , the dose value was up to $9 \%$ more than the measured value at $6 \mathrm{MV}$ energy [7]. Likewise, the study of Asnaashari et al., showed that in the lung tissue, EPL and ETAR algorithms calculated dose were $8.7 \%$ and $7.8 \%$ more than the measured values [32]. However, in both algorithms for the points within the lung tissue outside of the radiation field (point 9 in case 1), the calculated values were less than the measured values due to overlooking the lateral scattering of the electrons. The difference between calculation and measurement in the EPL algorithm was greater than in the ETAR algorithm.

At $6 \mathrm{MV}$ energy, both the EPL and the ETAR algorithms calculated the doses in the bone area inside the field in acceptable range (the dose values at point 10 in test 1 and in the first and third fields of test 4 were in the acceptable error range). However, both algorithms showed that the dose value in bone area below the shield was significantly less than the measured value; the EPL algorithm's error in this case was more than the ETAR algorithm's error. This test suggested that both algorithms estimated the dose values, resulting from scattering in the bone tissue (when bone was shielded), less than the measured value. Asnaashari et al., indicated that correction-based algorithms in bone tissue showed doses of $5-6$ $\%$ less than the measured values [32].

In assessing $18 \mathrm{MV}$ energy just like $6 \mathrm{MV}$ energy, test 4 showed that in low-density tissues at shallow depths, the EPL algorithm had more errors than the ETAR algorithm. However, by increasing the depth, the error of EPL algorithm was less than that of ETAR algorithm 
and difference between the calculation and the measurement decreased; this reduction was less for the ETAR algorithm. In fact, the method of calculating the dose in the ETAR algorithm was less dependent on the depth comparing with EPL algorithm. At $18 \mathrm{MV}$ energy, for the points, which were outside the field and within the lung tissue, the calculated values were less than the measured values. Although both algorithms calculated the dose values less than the measured values for the points outside the field and within the lung tissue, it was observed that the ETAR algorithm calculated the values with smaller error. The greater difference between measured value and calculated value in the field with gantry angle of $180^{\circ}$, in comparison with the field with gantry angle of $0^{\circ}$, was due to the heterogeneity of bone tissue in radiation path in the field of $180^{\circ}$. In the field with gantry angle of $0^{\circ}$, there was no heterogeneity of bone tissue in the beam path. However, for lung tissue located outside the beam path, the calculated dose value was estimated to be less than the measured value. At $18 \mathrm{MV}$ energy, both algorithms calculate the dose value in bone tissue less than the measured value. Besides, by increasing the field size, the difference between the measurement and calculation in bone tissue (point 10) increased. This discrepancy was higher in the EPL algorithm than in the ETAR algorithm. In addition, the greatest difference between the calculation and measurement for bone tissue was when the tissue was outside the radiation field. Other studies also demonstrated that the calculated doses in the bone tissue were less than the measured values $[7,32]$.

\section{Conclusion}

Each treatment-planning software contains some errors in the dose calculation. These errors are more found in heterogeneous areas. It is recommended that for each set of radiation data and each algorithm, precise examinations be made regarding the accuracy of dose calculations. Knowing the error of dose calcula- tion in each treatment planning system will provide the responsible physician and physicist with an accurate estimation of the actual dose delivered to the tumor. In this empirical study, the TEC-DOC 1583 practical guideline presented by IAEA was used. Experimental measurements were obtained using the ionization chamber at the intended points based on the abovementioned guideline at energies of $6 \mathrm{MV}$ and $18 \mathrm{MV}$. The values obtained from the measurements were compared with the values obtained from the calculations of EPL and ETAR algorithms. In the EPL algorithm compared with the ETAR algorithm, more points were found to be in line with the agreement criteria, while the numerical values of the errors in ETAR algorithm were 1 - 2\% less than that of EPL algorithm. The biggest error in calculating was when the calculations were carried out in the lung heterogeneity with low density or inside the bone tissue with high density and in the low depth. Moreover, the sum of errors was higher in high energy than low energy.

\section{Acknowledgment}

This study was extracted from a $\mathrm{PhD}$ thesis by Azizollah Rahimi and supported financially by the Research Affairs of Ahvaz Jundishapur University of Medical Sciences, Ahvaz, Iran (Grant number: U-96009).

\section{Conflict of Interest}

None

\section{References}

1. Herrick AC. A comparative dosimetric analysis of the effect of heterogeneity corrections used in three treatment planning algorithms. Electronic Thesis or Dissertation; University of Toledo; 2010. Available from: https://etd.ohiolink.edu/.

2. Ahnesjo A, Aspradakis MM. Dose calculations for external photon beams in radiotherapy. Phys Med Biol. 1999;44:R99-155. PubMed PMID: 10588277.

3. Woon YL, Heng S, Wong J, Ung NM. Comparison of selected dose calculation algorithms in radiotherapy treatment planning for tissues with inhomogeneities. J Phys: Conf Ser. 2016;694(1):1-5. 
Accuracy Evaluation of Algorithms in TPS

doi: 10.1088/1742-6596/694/1/012024.

4. Krieger T, Sauer OA. Monte Carlo- versus pencil-beam-/collapsed-cone-dose calculation in a heterogeneous multi-layer phantom. Phys Med Biol. 2005;50:859-68. doi: 10.1088/00319155/50/5/010. PubMed PMID: 15798260.

5. Zhu XR, Low DA, Harms WB, Purdy JA. A convolution-adapted ratio-TAR algorithm for 3D photon beam treatment planning. Med Phys. 1995;22:1315-27. doi: 10.1118/1.597516. PubMed PMID: 7476719.

6. Yorke E, Harisiadis L, Wessels B, Aghdam H, Altemus $\mathrm{R}$. Dosimetric considerations in radiation therapy of coin lesions of the lung. Int J Radiat Oncol Biol Phys. 1996;34:481-7. doi: 10.1016/03603016(95)02036-5. PubMed PMID: 8567352.

7. Rutonjski L, Petrovic B, Baucal M, Teodorovic M, Cudic O, Gershkevitsh E, et al. Dosimetric verification of radiotherapy treatment planning systems in Serbia: national audit. Radiat Oncol. 2012;7:155. doi: 10.1186/1748-717X-7-155. PubMed PMID: 22971539. PubMed PMCID: PMC3504524.

8. Orton CG, Chungbin S, Klein EE, Gillin MT, Schultheiss TE, Sause WT. Study of lung density corrections in a clinical trial (RTOG 88-08). Radiation Therapy Oncology Group. Int J Radiat Oncol Biol Phys. 1998;41:787-94. doi: 10.1016/s03603016(98)00117-5. PubMed PMID: 9652839.

9. Lopes MC, Cavaco A, Jacob K, Madureira L, Germano S, Faustino S, et al. Treatment planning systems dosimetry auditing project in Portugal. Phys Med. 2014;30:96-103. doi: 10.1016/j. ejmp.2013.03.008. PubMed PMID: 23623589.

10. Klein EE, Morrison A, Purdy JA, Graham MV, Matthews J. A volumetric study of measurements and calculations of lung density corrections for 6 and $18 \mathrm{MV}$ photons. Int $J$ Radiat Oncol Biol Phys. 1997;37:1163-70. doi: 10.1016/s03603016(97)00110-7. PubMed PMID: 9169827.

11. Gershkevitsh E, Schmidt R, Velez G, Miller D, Korf $E$, Yip F, et al. Dosimetric verification of radiotherapy treatment planning systems: results of IAEA pilot study. Radiother Oncol. 2008;89:338-46. doi: 10.1016/j.radonc.2008.07.007. PubMed PMID: 18701178.

12. Engelsman M, Damen EM, Koken PW, Van't Veld AA, Van Ingen KM, Mijnheer BJ. Impact of simple tissue inhomogeneity correction algorithms on conformal radiotherapy of lung tumours. Radiother Oncol. 2001;60:299-309. PubMed PMID: 11514010.

13. El-Khatib EE, Evans M, Pla M, Cunningham JR. Evaluation of lung dose correction methods for photon irradiations of thorax phantoms. Int $\mathrm{J}$ Radiat Oncol Biol Phys. 1989;17:871-8. doi: 10.1016/0360-3016(89)90081-3. PubMed PMID: 2777679.

14. De Jaeger K, Hoogeman MS, Engelsman M, Seppenwoolde $\mathrm{Y}$, Damen EM, Mijnheer BJ, et al. Incorporating an improved dose-calculation algorithm in conformal radiotherapy of lung cancer: re-evaluation of dose in normal lung tissue. Radiother Oncol. 2003;69:1-10. doi: 10.1016/s01678140(03)00195-6. PubMed PMID: 14597351.

15. Alam R, Ibbott GS, Pourang R, Nath R. Application of AAPM Radiation Therapy Committee Task Group 23 test package for comparison of two treatment planning systems for photon external beam radiotherapy. Med Phys. 1997;24:2043-54. doi: 10.1118/1.598119. PubMed PMID: 9434989.

16. Aarup LR, Nahum AE, Zacharatou C, Juhler-Nottrup $T$, Knoos T, Nystrom H, et al. The effect of different lung densities on the accuracy of various radiotherapy dose calculation methods: implications for tumour coverage. Radiother Oncol. 2009;91:40514. doi: 10.1016/j.radonc.2009.01.008. PubMed PMID: 19297051.

17. Meredith WJ, Neary G. The production of isodose curves and the calculation of energy absorption from standard depth dose data. The British Journal of Radiology. 1944;17:75-82. doi: 10.1259/00071285-17-195-75.

18. Liu Q, Liang J, Stanhope CW, Yan D. The effect of density variation on photon dose calculation and its impact on intensity modulated radiotherapy and stereotactic body radiotherapy. Med Phys. 2016;43:5717. doi: 10.1118/1.4963207. PubMed PMID: 27782711.

19. Breitman K, Rathee S, Newcomb C, Murray B, Robinson $D$, Field $C$, et al. Experimental validation of the Eclipse AAA algorithm. J Appl Clin Med Phys. 2007;8:76-92. doi: 10.1120/jacmp. v8i2.2350. PubMed PMID: 17592457. PubMed PMCID: PMC5722411.

20. Craig J, Oliver M, Gladwish A, Mulligan M, Chen J, Wong E. Commissioning a fast Monte Carlo dose calculation algorithm for lung cancer treatment planning. J Appl Clin Med Phys. 2008;9:2702. PubMed PMID: 18714276. PubMed PMCID: PMC5721711.

21. International Atomic Energy Agency. Commissioning of radiotherapy treatment planning systems: Testing for typical external beam treatment techniques. IAEA Tecdoc Series No. 1583; Vienna: IAEA; 2008. p. 1-67.

22. Batho HF. Lung Corrections in Cobalt 60 Beam 
Therapy. J Can Assoc Radiol. 1964;15:79-83. PubMed PMID: 14173312.

23. Sontag MR, Cunningham JR. Clinical application of a CT based treatment planning system. Comput Tomogr. 1978;2:117-30. doi: 10.1016/03638235(78)90009-1. PubMed PMID: 699542.

24. Sontag MR, Cunningham JR. The equivalent tissue-air ratio method for making absorbed dose calculations in a heterogeneous medium. Radiology. 1978;129:787-94. doi: 10.1148/129.3.787. PubMed PMID: 725060.

25. Purdy JA. Photon Dose Calculations for ThreeDimensional Radiation Treatment Planning. Semin Radiat Oncol. 1992;2:235-45. doi: 10.1053/ SRA000200235. PubMed PMID: 10717040.

26. Cunningham JR. Scatter-air ratios. Phys Med Biol. 1972;17:42-51. PubMed PMID: 5071500.

27. Greene D, Stewart JG. Isodose Curves in Non-Uniform Phantoms. Br J Radiol. 1965;38:378-85. doi: 10.1259/0007-1285-38-449-378. PubMed PMID: 14280292.

28. Purdy J, Prasad S. Current methods and algorithms in radiation absorbed dose calculation and the role of computed tomography: A review. United States; Raven Press Publications; 1983.

29. Sundbom L. Dose planning for irradiation of thorax with 60-Co in fixed-beam teletherapy. Acta Radiol Ther Phys Biol. 1965;3:342-52. doi: 10.3109/02841866509133109. PubMed PMID: 5838014.

30. O'Connor JE. The variation of scattered x-rays with density in an irradiated body. Phys Med Biol. 1957;1:352-69. doi: 10.1088/0031-9155/1/4/305. PubMed PMID: 13452841.

31. International Atomic Energy Agency. Specification and acceptance testing of radiotherapy treatment planning systems. IAEA Tecdoc Series No. 1540; Vienna, Austria: IAEA; 2007.

32. Asnaashari K, Nodehi MR, Mahdavi SR, Gholami S, Khosravi HR. Dosimetric comparison of different inhomogeneity correction algorithms for external photon beam dose calculations. $J$ Med Phys. 2013;38:74-81. doi: 10.4103/0971-6203.111310. PubMed PMID: 23776310. PubMed PMCID: PMC3683304. 